\title{
Dionis: A novel remote-center-of-motion parallel manipulator for Minimally Invasive Surgery
}

\author{
R. Beira*, L. Santos-Carreras, G. Rognini, H. Bleuler and R. Clavel \\ Laboratoire de Systèmes Robotiques, École Polytechnique Fédérale de Lausanne (EPFL), \\ Lausanne, Switzerland
}

\begin{abstract}
The large volume and reduced dexterity of current surgical robotic systems are factors that restrict their effective performance. To improve the usefulness of surgical robots in minimally invasive surgery (MIS), a compact and accurate positioning mechanism, named Dionis, is proposed in this paper. This spatial hybrid mechanism based on a novel parallel kinematics is able to provide three rotations and one translation for single port procedures. The corresponding axes intersect at a remote center of rotation (RCM) that is the MIS entry port. Another important feature of the proposed positioning manipulator is that it can be placed below the operating table plane, allowing a quick and direct access to the patient, without removing the robotic system. This, besides saving precious space in the operating room, may improve safety over existing solutions. The conceptual design of Dionis is presented in this paper. Solutions for the inverse and direct kinematics are developed, as well as the analytical workspace and singularity analysis. Due to its unique design and kinematics, the proposed mechanism is highly compact, stiff and its dexterity fullfils the workspace specifications for MIS procedures.
\end{abstract}

Keywords: Minimally invasive surgery, surgical robot, parallel mechanism, kinematic analysis, workspace analysis

\section{Introduction}

A major progress in abdominal surgery has occurred during the last decades with the introduction of laparoscopic and minimally invasive techniques. These innovative procedures focused much attention due to their several advantages: Smaller abdominal incisions needed, resulting in faster recovery of the patient, improved cosmetics, and shorter stay in the hospital. The safety, efficiency and cost-effectiveness of laparoscopic surgery have subsequently been demonstrated in clinical trials for many routine abdominal opera-

${ }^{*}$ Corresponding author. E-mail: ricardo.beira@epfl.ch. tions [2, 27]. However, from the surgeon's point of view there are still many difficulties in learning and performing such procedures with current laparoscopic equipment, which is non-ergonomic, non-intuitive and lacking in adequate visual and tactile feedback [4, 18].

In order to overcome the disadvantages of traditional minimally invasive surgery (MIS), robot technology has been introduced into the operation room. Although a wide range of diagnostic and therapeutic robotic devices have been developed [29] and [34], the only commercial systems that have already been used in human surgery are the da Vinci System, by Intuitive Surgical [25], and ZEUS, by Computer Motion. Following the fusion between the two companies, the ZEUS robot is no longer produced. 
Despite various existing interesting systems and after more than ten years of robotic MIS research, surgical robotics is still only at the very beginning of a very promising large scale development. One of the major open drawbacks is that the current surgical robots are voluminous, competing for precious space within the operating room (OR) environment and significantly increasing OR preparation time. Access to the patient is thus impaired and this raises safety concerns. In addition, robotic systems offer excellent vision and precise tissue manipulation within a defined area but they are limited in operations involving more than one quadrant of the abdomen. Since many gastrointestinal operations involve operating in at least two abdominal quadrants, the repeated disconnection and movement of the robots increase significantly the duration of the surgical procedure.

Another drawback of current surgical systems is related to the mechanisms that hold and place the surgical instruments into the abdomen, remaining external to the patient. Their access within the abdomen is limited since the instruments are constrained by the abdominal wall at their point of entry. They are also further restricted by this fulcrum effect, due to the fact that their internal motion is mirrored and magnified outside the body by the robotic arms. Moreover, surgical instruments have limited space to move, leading to eventual collisions. Consequently, a key feature of most surgical robot systems is the remoter center of rotation (RCM), that allows the manipulated tool to pivot around a fixed point, coincident with the insertion point of the tool through the patient's abdomen. Although some robotic systems use a passive RCM [8, 29], or a virtually constrained RCM [19], a mechanically constrained RCM is often considered safer.

There is a variety of ways to achieve a mechanically constrained RCM [5, 22, 32, 33]. For those spherical mechanisms consisting of revolute joints, all the rotation axes intersect at the center of the mechanism, effectively meeting the pivot constraint of MIS. The implementation of this approach can be done using parallelogram linkages $[28,30]$, or other linkage configuration [17, 23]. However, in most cases, actuators are directly mounted at the joints. This may result in: (1) addition of inertial loads that adversely affect system performance $[6,7]$ or (2) lack of DOFs to place and guide the MIS instruments within the abdominal cavity [23, 24].

In order to overcome some of these problems, our research group is working on a novel surgical robotic platform aiming to bring precise bi-manual manipulation and standard surgical procedures inside the abdominal cavity. The overall platform is mainly composed by two subsystems. One of them is an endoscopic unit, which is a cable actuated micromanipulator system, introducing a higher degree of dexterity, payload capacity, stiffness and precision to MIS procedures. The other subsystem, which is presented in this paper, is a novel external positioning manipulator, able to provide sufficient dexterity and precision to position the endoscopic unit or a conventional MIS instrument. The unique design of the proposed system permits to keep the above-mentioned characteristics at any location within the abdominal cavity. Extensive discussions with the medical community have provided a precious input to establish a highly innovative engineering surgical system. The proposed manipulator design will contribute to increase the precision and stability of abdominal surgical procedures, increasing their reliability. This is possible taking into account the performance of its parallel structure.

The organization of the paper is summarized henceforward. After a short description of the related geometry and constraints, presented on Section 2, manipulator's mobility is analysed in Section 3 and both inverse and direct kinematics are solved in closedform for the parallel structure that forms Dionis main body, in Section 4. In Section 5, a symbolic form for the Jacobian matrix is calculated and the singular configurations are identified in Section 6. After that, a study of the workspace is performed in Section 7. Conclusions and outlook are presented in Section 8.

\section{System overview}

\subsection{Surgical procedure}

Recent developments in surgery show a clear trend toward less invasive methods of access over the past decades. While conventional laparoscopy is the standard treatment for various disorders at present, newer methods such as single incision laparoscopy (SIL) and Natural Orifice Translumenal Endoscopic Surgery (NOTES) are gaining clinical significance worldwide $[9,15]$. Besides hypothetical clinical advantages such as faster recovery, fewer pain medication and milder anaesthesia, surveys point out that potential patients actually favour cosmetically superior surgical approaches [20]. While pure NOTES will deliver 
cosmetically perfect outcomes with no external scars, this method is still under development and only performed at a few hospital centers world-wide [13, 36]. SIL, on the other hand, is a feasible technique that has gained large attention during the last few years, reporting many successful cases on medical publications [11, 12]. The great majority of single incision laparoscopy currently performed, is carried out through the umbilicus with penetration of the abdominal wall, by the umbilical midline. However, such an approach might lead to a deformed umbilicus, whose integrity and appearance is considered to be extremely important for many patients $[3,14]$, and to an increased rate of incisional hernias after the procedure [26].

Due to these presumed disadvantages, a novel approach to enter into the abdominal cavity for SIL has been developed and may represent a bridge between the cosmetic advantages of NOTES with the technical feasibility of SIL. Subcutaneous surgical tunneling (SST) disconnects the skin incision from the entrance of the peritoneal cavity through the abdominal wall [21]. Therefore, the skin incision can be placed in almost any cosmetically favorable location of the body such as the supra-pubic hair, groin, axillae or previous (Fig. 1). Then, a subcutaneous tunnel is formed to enter the peritoneal cavity through the abdominal wall at a mechanically favorable location, such as the rectal muscle to decrease the risk of incisional hernias.

The flexibility of this method of access makes it possible to theoretically customize each incision to the specifics of each patient, regarding existing scars, cosmetic preferences and individual weak areas of the

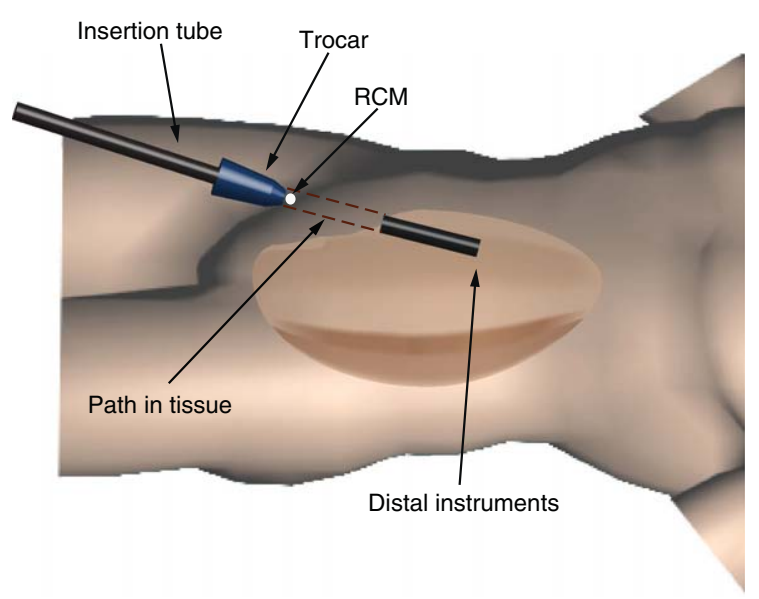

Fig. 1. Subcutaneous surgical tunnelling procedure. abdominal wall. In addition, having the MIS instruments inserted close to a horizontal orientation allows the access to different quadrants of the abdomen, avoiding the docking and disengagement of the robot and decreasing significantly the total operating time (Fig. 1). However, the mobility of the surgical instruments may be lower in the case of SST than in a conventional SIL procedure.

\subsection{The complete platform}

To the best of the authors' knowledge, the design concept followed in this work consists of one of the first attempts to develop a robotic platform aiming to perform surgical procedures by SST. The idea is to bring bi-manual manipulation and standard surgical procedures inside the abdominal cavity, with a cable actuated micro-robotic system, stabilized by an external system and inserted through an incision on the supra-pubic hair region, Fig. 2.

The surgical platform proposed in Fig. 3 is mainly composed by two subsystems: (1) an external positioning unit, the Dionis manipulator, and (2) an endoscopic unit. A newly designed micro-manipulator system operates to increase the degree of dexterity, payload capacity, stiffness and precision inside the patient's body.

The purpose of the Dionis manipulator is to position the micro-manipulators of the endoscopic unit inside the human body, without violating the constraints imposed by the fixed tissue incision point. In this way, the proposed external manipulator pro-

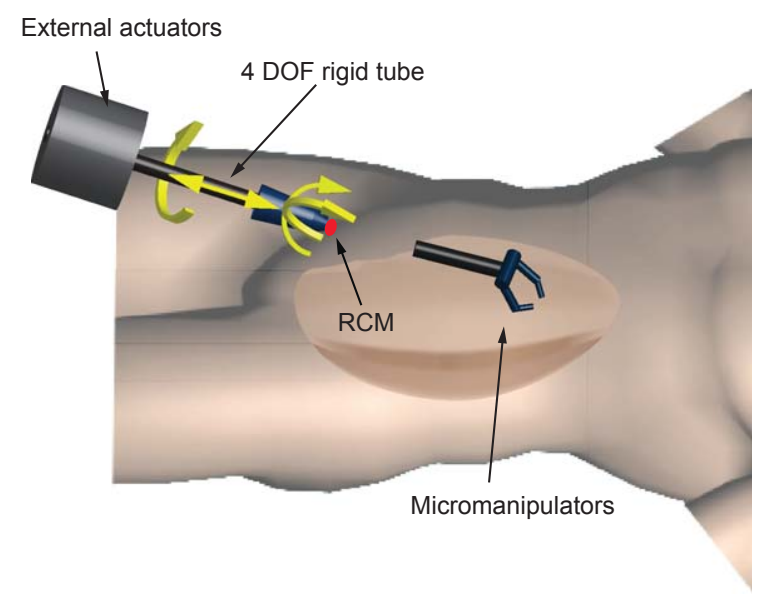

Fig. 2. Conceptual representation of the surgical platform. 


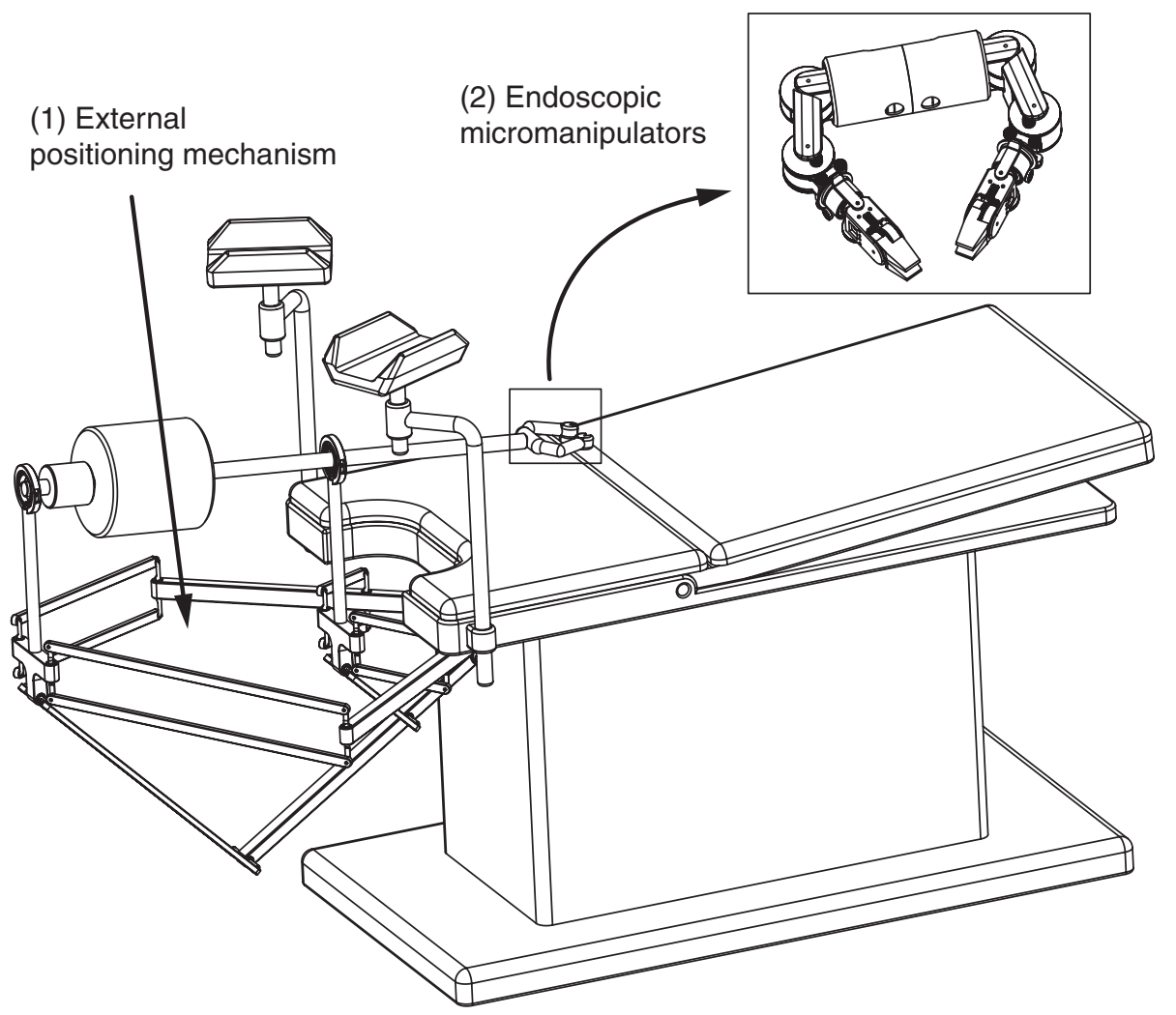

Fig. 3. Conceptual design of the complete surgical platform.

vides 4 DOF, with a fixed RCM, for positioning the endoscopic micro-manipulators inside the abdominal cavity. The related kinematics gives to the insertion tube (IT) two rotary degrees of freedom about the incision port, placed around the supra-pubic-hair area, plus a linear movement in the direction of the same point, along the axis of the IT. The fourth DOF is a rotation about the IT's axis, given by a fourth actuator of the external unit, Fig. 4.

Since the external manipulator cannot provide the desired mobility needed to perform complicated surgical procedures, the extra DOFs are given by two micro-manipulators. In order to be as intuitive to control as possible, the degrees of freedom are designed to resemble a simplified human arm. Two micromanipulators are used to perform actions such as pulling and cutting the tissue or eventually suturing. The stereoscopic camera will be located between the two arms, providing eye-manipulator alignment similar to human eye-hand alignment, thus enhancing the telepresence and intuitiveness of the system. This gives the impression to the surgeon that he is operating inside the patient's body with his own two hands.

\subsection{External manipulator geometry: The Dionis novel parallel structure}

Despite showing good operating characteristics (large workspace, high flexibility and dexterity), serial manipulators present disadvantages such as low precision, low stiffness and low payload. On the other hand, parallel kinematic manipulators offer essential advantages, mainly related to lower moving masses, higher rigidity and payload-to-weight ratio, higher natural frequencies, better accuracy, simpler modular mechanical construction and possibility to locate actuators on the fixed base. These characteristics make parallel manipulators extremely suitable for surgical applications. Taking into account that stiffness and precision are considered to be key features on external positioning mechanisms for MIS, the proposed manipulator is 


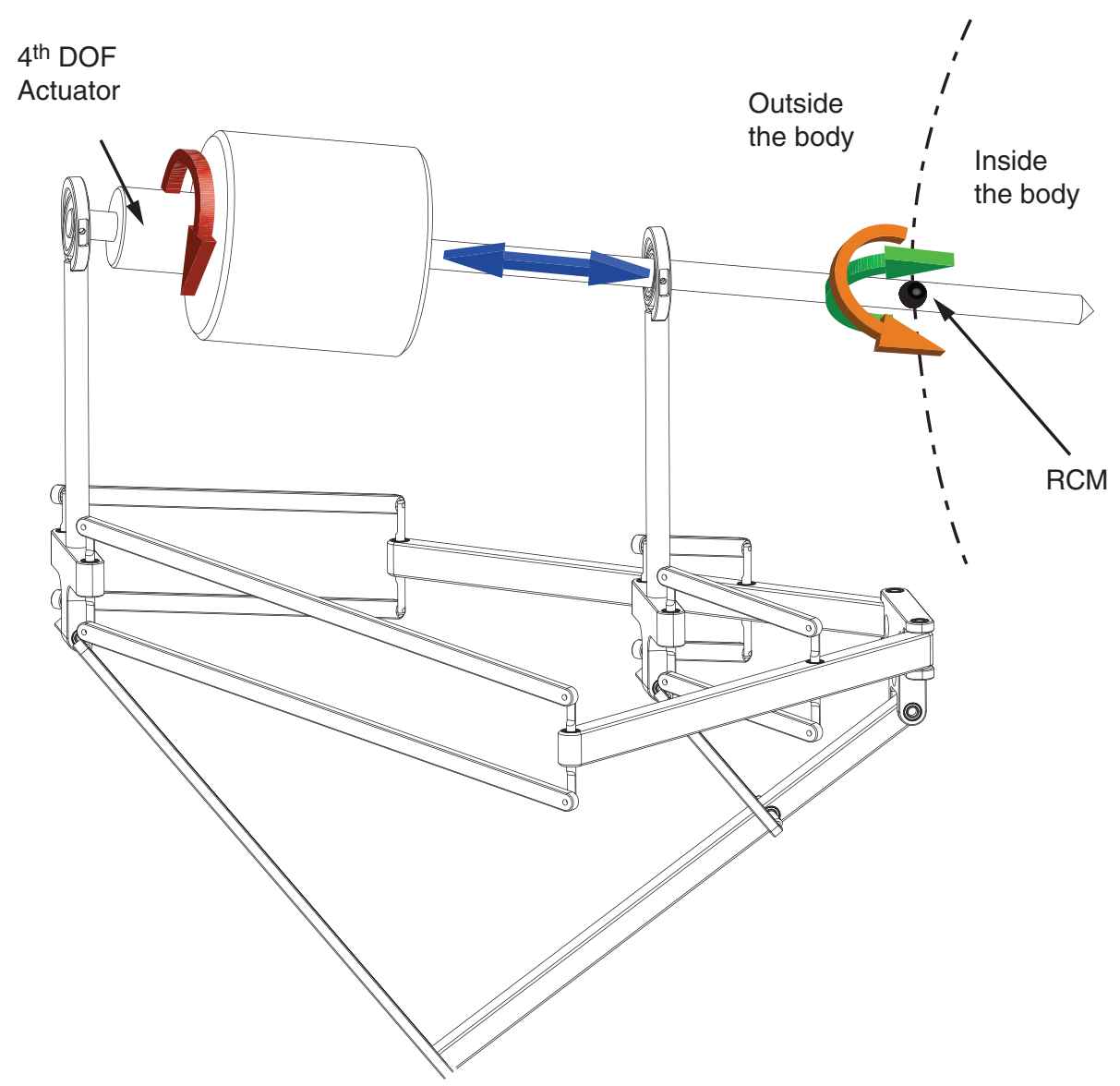

Fig. 4. Degrees of freedom of the external manipulator.

based on a parallel kinematics, to reproduce the needed degrees of freedom.

A schematic of the proposed manipulator is shown in Fig. 5. The RCM, point $\mathrm{O}$, is placed on the $x$-axis of the fixed reference frame, $F(x, y, z)$, and is distant by an offset $t$ from the origin, $\mathrm{O}^{\prime}$, which is placed in the intersection of lines $t_{1}, t_{2}$ and $t_{3}$, that belong to the stationary platform, $P_{S}$, in the XY plane. In addition, lines $t_{1}, t_{2}$ and $t_{3}$ are perpendicular to axes $a_{11}, a_{12} a_{13}$, respectively. Three identical limbs connect the moving platforms, $P_{M}$ and $P_{I}$, to the stationary platform. Each limb consists of an input link, directly connected to the actuator, placed on $P_{S}$ and two driven links, connected to $P_{M}$ and $P_{I}$. The input links are labeled $D_{11}, D_{12}$, and $D_{13}$ and have length $d_{1}$. The driven links are composed by planar four-bar parallelograms, $D_{21}, D_{22}, D_{23}, D_{21}^{\prime}$, $D_{22}^{\prime}$ and $D_{23}^{\prime}$ and have length $d_{2}$ and $d_{2}^{\prime}$ respectively. All of the links and platforms are considered as rigid bodies (Fig. 5).
The $n$th limb of the manipulator is shown in Fig. 6. In each limb, the driven links, the input link, and the three platforms are connected by four parallel revolute joints, at axes $a_{1 n}, a_{2 n}, a_{3 n}, a_{2 n}^{\prime}$ and $a_{3 n}^{\prime}$ that are perpendicular to the axes of the four-bar parallelogram for each limb. A coordinate system, $L_{n}\left(u_{n}, v_{n}, w_{n}\right)$, is attached to the fixed base, $P_{S}$, in the actuated joint of each limb, such as the $u_{n}$ axis is perpendicular to the axis of rotation of the joint, $a_{1 n}$, and at an angle $\vartheta_{n}$ from the $x$-axis, while being in the plane of $P_{S}$. The $v_{n}$-axis is along $a_{1 n}$.

The actuation angle, $\alpha_{n}$, for the $n$th limb, defines the angular orientation of the input link relative to the XY plane, on platform $P_{S}$. Vectors $\mathbf{m}$ and $\mathbf{e}$ are respectively the position vectors of points $\mathrm{M}$ and $\mathrm{E}$, in the F coordinate frame. $\mathrm{M}$ and I are placed at the center of circles $c_{M}$ and $c_{I}$ of radius $r_{M}$ and $r_{I}$, that belong to platforms $P_{M}$ and $P_{I}$. Vector $\mathbf{l}$ is aligned with the output link, $L_{E}$, from point $\mathrm{M}$ to point E. Angles $\beta_{n}$ 


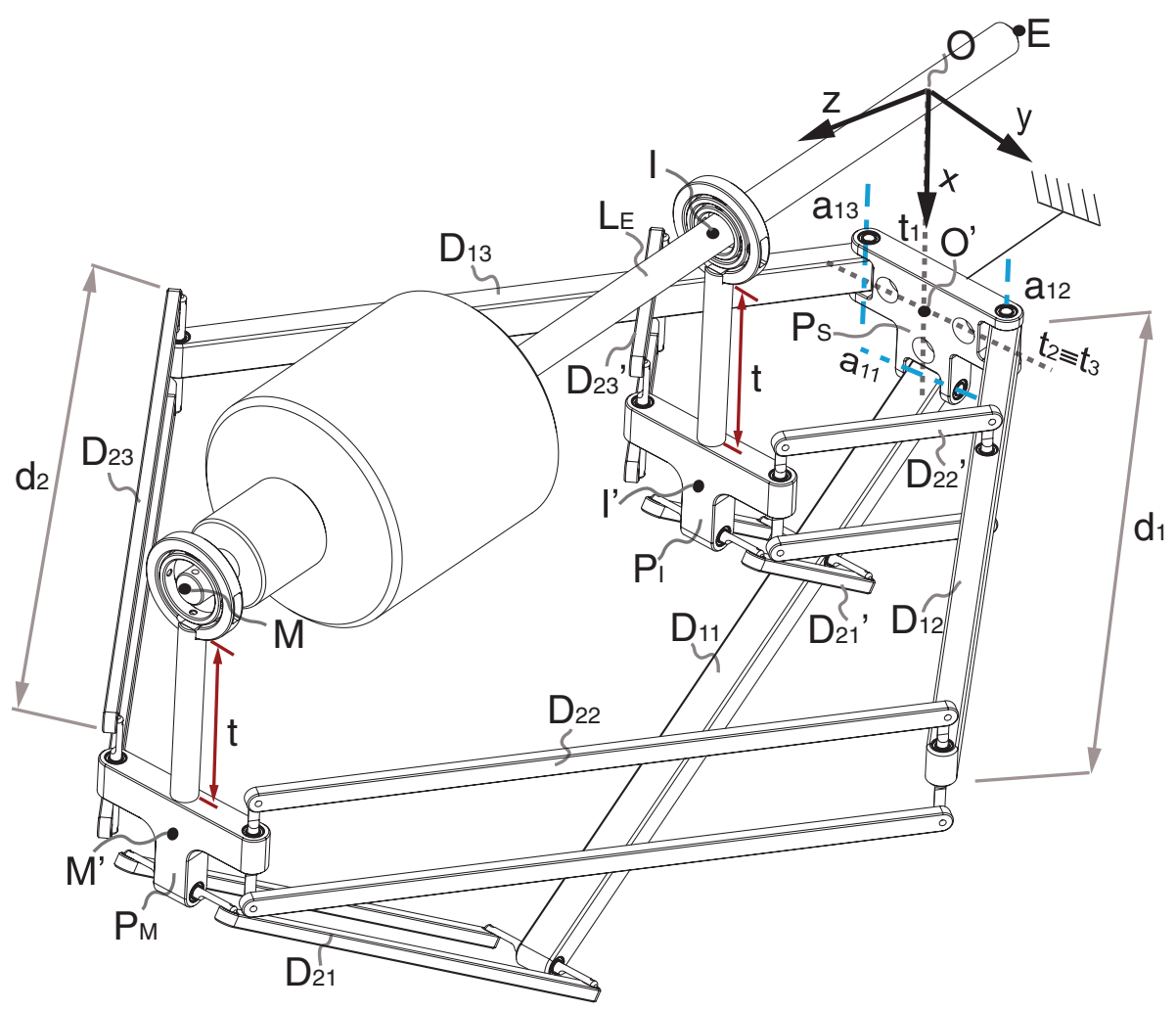

Fig. 5. Dionis schematics.

and $\beta_{n}^{\prime}$ are defined from the direction of input links, axis $d_{1 n}$, to the direction of the plane containing the parallelograms of driven links, $d_{2 n}$ and $d_{2 n}^{\prime}$. Angles $\gamma_{n}$ and $\gamma_{n}^{\prime}$ are defined by the angles from the directions of the driven links, $d_{2 n}$ and $d_{2 n}^{\prime}$, through axis $a_{2 n}$ and $a_{2 n}^{\prime}$

The configuration of the limbs is based on the well known Delta robot [10]. It is in fact composed by a pair of 3 four-bar-parallelogram-links fixed on the same input links. Therefore, the two platforms (the intermediate, $P_{I}$, and the distal one, $\left.P_{M}\right)$ move in the same manner except that $P_{M}$ moves with bigger ranges than $P_{I}$. Link, $L_{E}$, containing the end-effector, E, is then connected to points $\mathrm{M}$ and $\mathrm{I}$ by an universal joint and a sliding spherical joint respectively. The output of the proposed design results in: Two rotations of $L_{E}$ around the $\mathrm{X}$ and $\mathrm{Y}$ axis, and a translation of $\mathrm{E}$ on the direction $\overline{M O}$.

To guarantee a perfect RCM, a geometrical ratio is needed. This ratio is based on the Intercept Theorem [1], which states that: If two or more parallel lines are intersected by two self intersecting lines, then the ratios of the line segments of the first intersecting line is equal to the ratio of the similar line segments of the second intersecting line. In other words, and for the example of Fig. 7:

$$
\frac{\overline{S D}}{\overline{C D}}=\frac{\overline{S B}}{\overline{A B}}
$$

On Fig. 8(a), a simplified 2D representation of the Dionis ${ }^{1}$ is shown. The upper limb ("dashed") is virtually rotated $\pi \mathrm{rad}$ from the one bellow, around the $\mathrm{Z}$ axis. According to the Delta principle [10], the rotations of the moving platforms are blocked and $P_{M}$ and $P_{I}$ are always parallel and vertical. Consequently, in

\footnotetext{
${ }^{1}$ It is the name of a 17 th century mathematician and astronomer, Achille Pierre Dionis, who studied, among other topics, the alignement of eclipses. The alignement of points $\mathrm{O}, \mathrm{I}$ and $\mathrm{M}$ is precisely the characterization of the new parallel kinematic structure.
} 


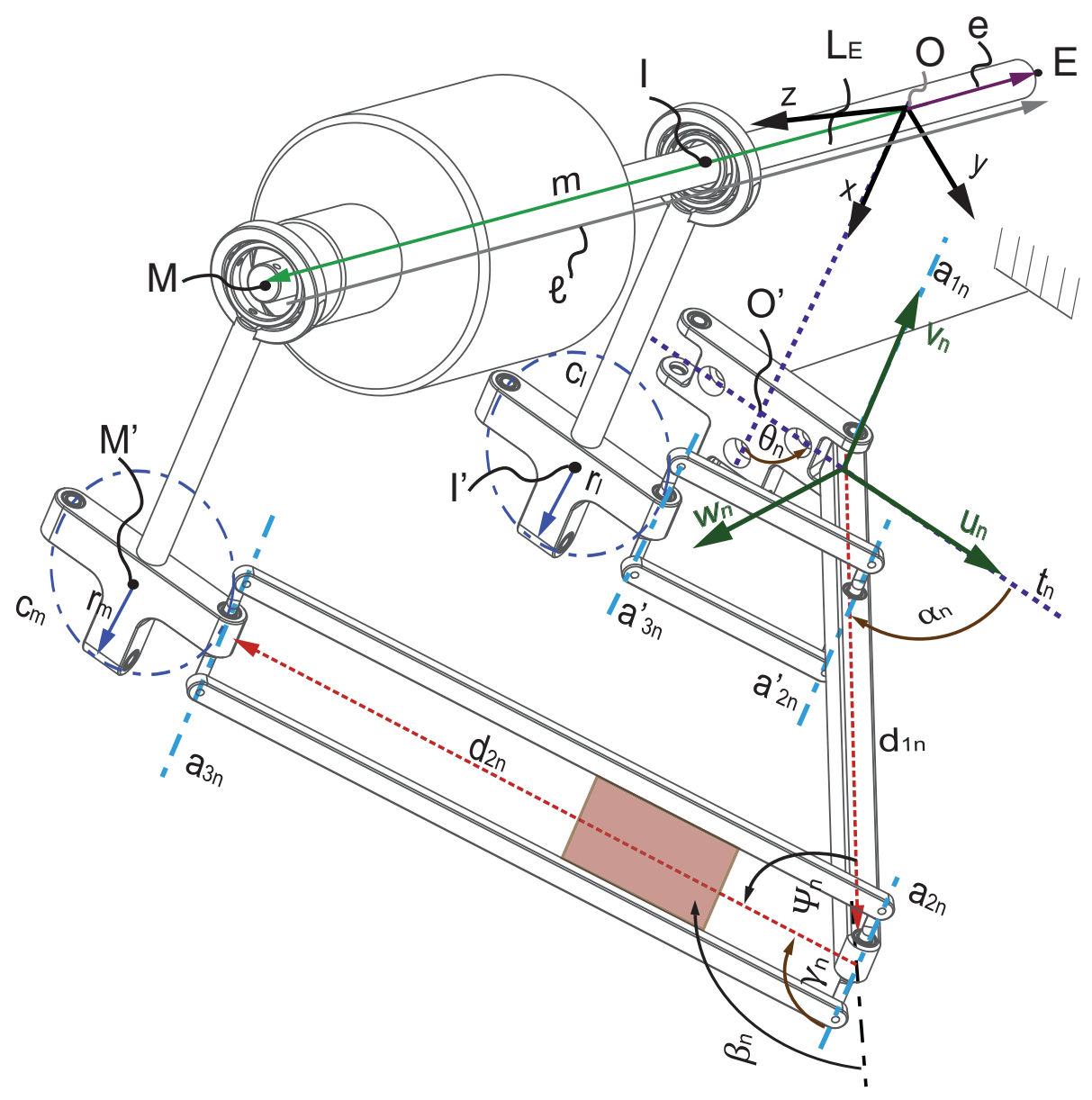

Fig. 6. Limb schematics.

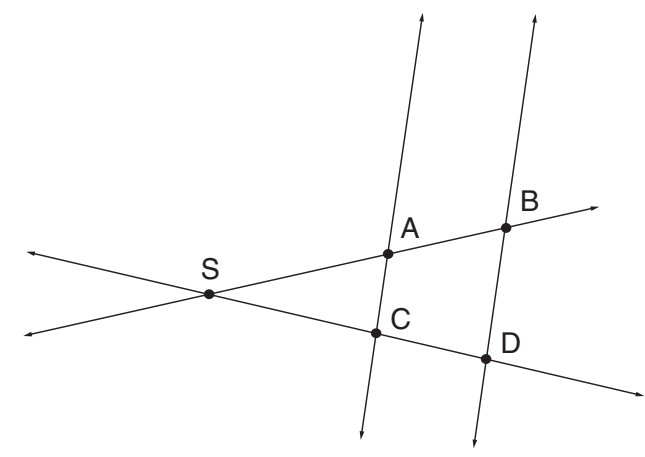

Fig. 7. Illustration of the Intercept Theorem.

order to have the link $\overline{M E}$ always aiming at the RCM, it is necessary to have points $\mathrm{A}, \mathrm{C}^{\prime}$ and $\mathrm{C}$ aligned. This is true if segments $\mathrm{B}^{\prime} \mathrm{C}^{\prime}$ and $\mathrm{BC}$ are parallel and if $B C / B^{\prime} C^{\prime}=A B / A B^{\prime}$ (Intercept Theorem). If these conditions are not fulfilled, the behavior of the robot will be similar but without a perfect RCM. By contrast, if they are satisfied, point I will always be aligned with $\mathrm{O}$ and $\mathrm{M}$, for any position of $\mathrm{M}$, and platform $P_{I}$ is passively moved to guarantee this configuration. According to the above mentioned constraints, a geometrical simplification can be made, assuming zero-size platforms, which significantly simplifies the kinematic analysis of the mechanical structure, Fig. 8(b). In addition, an equivalent architecture can be introduced, extending the platforms at $\mathrm{O}, \mathrm{I}$ and $\mathrm{M}$ by a distance $t$, as shown in Fig. 8(c). In this way, the $\mathrm{RCM}$, is translated by a distance $t$, in the platform's extension direction, resulting in a mechanism with the same kinematics.

It is also important to point out that this kinematics can also be applied in other configurations that are not specific to the surgical procedure presented in the 
(a)

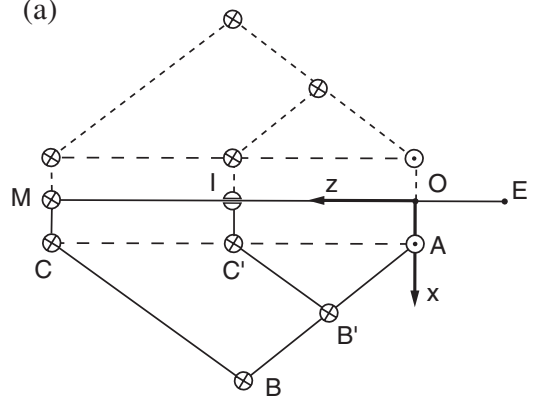

(b)

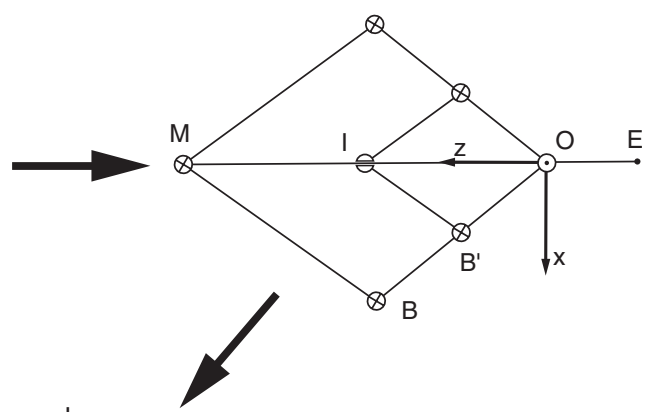

(c)

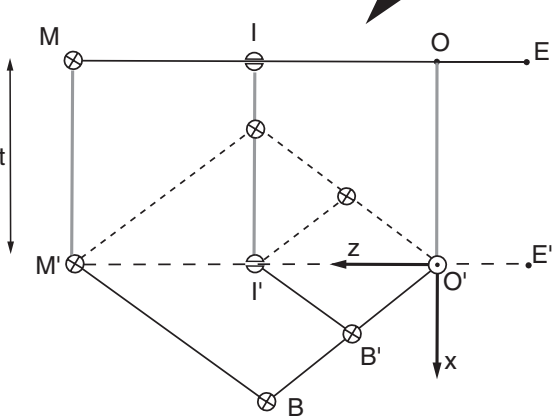

Fig. 8. 2D representation of Dionis manipulator.

previous section. Figure 9 shows two other possible configurations of the proposed kinematics.

\section{Manipulator mobility}

The proposed parallel platform hereafter is characterized by the kinematic structure shown in Fig. 10 . Considering the manipulator mobility, let $F$ be the degrees of freedom, $n$ the number of parts, $k$ the number of articulations, $f_{i}$ the degrees of freedom associated with the ith joint, and $\lambda=6$, the motion parameter. Then, the number of DOFs of a mechanism is determined by the Grübler-Kutzbach Criterion [31]:

$$
F=\lambda(n-k)+\sum_{i=1}^{j} f_{i}
$$

For the Dionis manipulator, we have: $n=13$ (3 inputs links, 6 driven links, 2 moving platforms, 1 slider-mount, 1 end-effector link); $k=18$ ( 3 actuated revolute joints, 1 spherical joint, 13 universal joint and 1 slider) and $\sum f_{i}=33$. Applying Eq. 2 to the Dionis manipulator results in: $F=3$, and consequently a mechanism with 3 DOF. The result would be the same considering all the bars of the parallelograms with a ball and a universal joints at each tip.

\section{Manipulator kinematics}

The kinematics of Delta-like Manipulators has been extensively studied by several authors $[10,31]$. Although they look similar in form, Dionis kinematics is simpler due to the dimensional constraints imposed by the Intercept Theorem as well as by the geometrically equivalent zero-sized platforms simplification (represented in Fig. 8). Although the RCM might not be completely stationary in a real prototype, due to a deficient production of the different components, in the following analysis it is assumed so, with the Intercept Theorem constraints perfectly fulfilled.

\subsection{Inverse kinematics}

The objective of the inverse kinematics solution is to define a mapping from the position of the end-effector, in a Cartesian space, to the set of joint angles that achieve that position. For this analysis, the position of point $\mathrm{E}\left(e_{x}, e_{y}, e_{z}\right)$ is considered known, and is given 


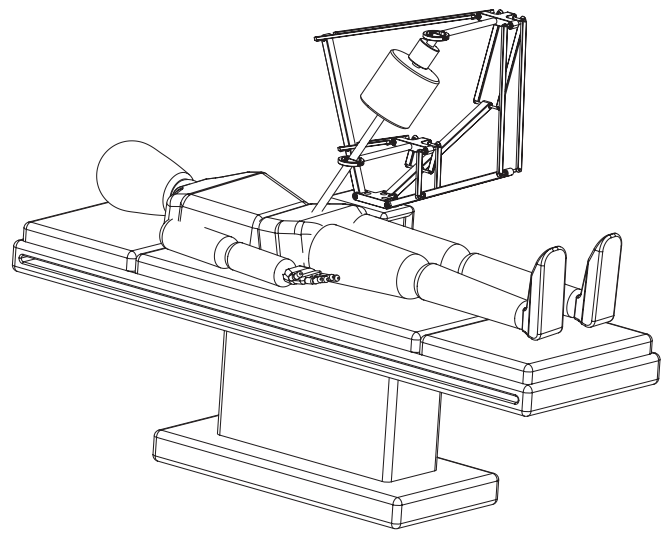

(a) Lateral position configuration

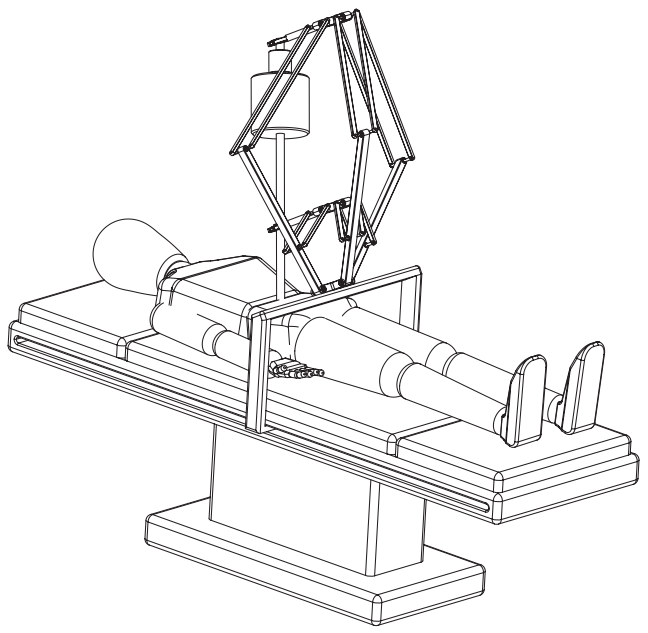

(b) Superior position configuration

Fig. 9. Example of potential working configurations for the Dionis manipulator

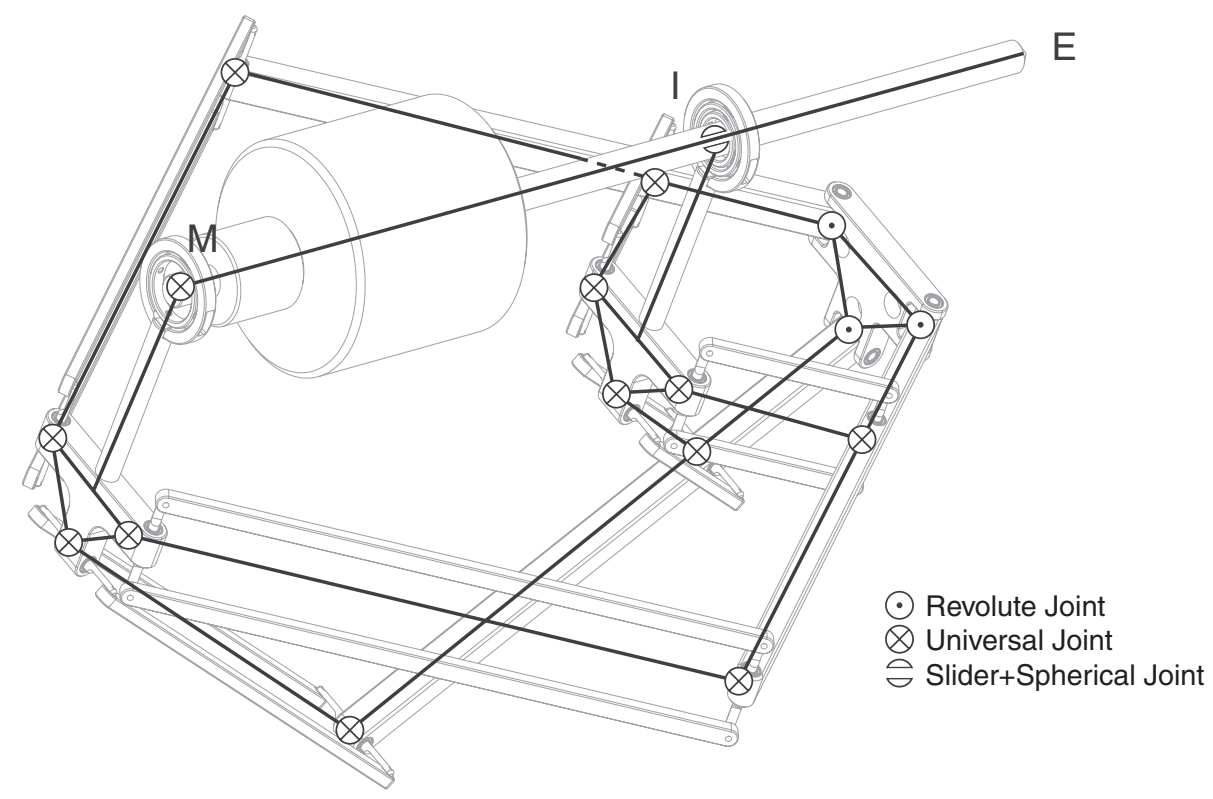

Fig. 10. Kinematic structure of Dionis manipulator.

by the position vector $\mathbf{e}$, which defines the location of $\mathrm{E}$ at the distal end of the output link in the $\mathrm{F}(x, y, z)$ coordinate frame. The inverse kinematics analysis produces the joint angles for each inputs link $\left(\alpha_{1}, \alpha_{2}\right.$ and $\alpha_{3}$ ) that yield the given location of the output point.
According to Fig. 6, the following relations for the $n$th limb can be written:

$$
\mathbf{d}_{1 n}+\mathbf{d}_{2 n}=\mathbf{m}=\mathbf{e}-\mathbf{l}
$$


where:

$$
\begin{gathered}
F \mathbf{e}=\left[\begin{array}{c}
e_{x} \\
e_{y} \\
e_{z}
\end{array}\right],{ }^{F} l=-\frac{\mathbf{e}}{\|\mathbf{e}\|}\|\mathbf{I}\|,{ }^{F} \mathbf{d}_{1 n}+{ }^{F} \mathbf{d}_{2 n} \\
={ }^{F} R_{L}\left({ }^{L_{n}} \mathbf{d}_{1 n}+{ }^{L_{n}} \mathbf{d}_{2 n}\right), \\
{ }^{F} R_{L}=\left[\begin{array}{ccc}
\cos \vartheta_{n}-\sin \vartheta_{n} & 0 \\
\sin \vartheta_{n} & \cos \vartheta_{n} & 0 \\
0 & 0 & 1
\end{array}\right], \\
{ }^{L_{n}} \mathbf{d}_{1 n}+{ }^{L_{n}} \mathbf{d}_{2 n}=\left[\begin{array}{cr}
d_{1} \cos \alpha_{n}+d_{2} \sin \gamma_{n} \cos \left(\alpha_{n}+\beta_{n}\right) \\
d_{2} \cos \gamma_{n} \\
d_{1} \sin \alpha_{n}+d_{2} \sin \gamma_{n} \sin \left(\alpha_{n}+\beta_{n}\right)
\end{array}\right] .
\end{gathered}
$$

The following system of three equations is obtained for Eq. 3 in the $L_{n}\left(u_{n}, v_{n}, w_{n}\right)$ coordinate frame.

$$
\left\{\begin{array}{l}
d_{1} \cos \alpha_{n}+d_{2} \sin \gamma_{n} \cos \left(\alpha_{n}+\beta_{n}\right)=\cos \vartheta_{n} m_{x} \\
\quad-\sin \vartheta_{n} m_{y} \\
d_{2} \cos \gamma_{n}=\sin \vartheta_{n} m_{x}-\cos \vartheta_{n} m_{y} \\
d_{1} \sin \alpha_{n}+d_{2} \sin \gamma_{n} \sin \left(\alpha_{n}+\beta_{n}\right)=m_{z}
\end{array}\right.
$$

and from Eq. 4:

$\gamma_{n}=\cos ^{-1}\left(\frac{\sin \vartheta_{n} m_{x}-\cos \vartheta_{n} m_{y}}{d_{2}}\right), \gamma_{n} \in[0, \pi]$

$\gamma_{n}, \beta_{n}$ may be determined taking into account the Carnot Theorem in the triangle formed by $\mathbf{m}, \mathbf{d}_{1}$ and $\mathbf{d}_{2}$,

$$
m^{2}=d_{1}^{2}+d_{2}^{2}-2 d_{1}^{2} d_{2}^{2} \cos \psi
$$

which is possible to develop in function of $\beta_{n}$ :

$$
\begin{gathered}
\beta_{n}=\cos ^{-1}\left(\frac{m_{x}^{2}+m_{y}^{2}+m_{z}^{2}-d_{1}^{2}-d_{2}^{2}}{2 d_{1}^{2} d_{2}^{2} \sin \gamma_{n}}\right), \\
\beta_{n} \in[0, \pi]
\end{gathered}
$$

Finally, with $\alpha_{n}$ and $\beta_{n}$ already calculated, an analytic expression of $\alpha_{n}$ can be obtained from Eq. 4 .

\subsection{Direct kinematics}

Direct kinematics solution defines a mapping from the known set of actuated joint angles to the unknown position of the moving platform. For the proposed manipulator, the joint angles that are considered known are those formed by the links and the fixed base of the manipulator $\left(\alpha_{1}, \alpha_{2}\right.$ and $\left.\alpha_{3}\right)$. The unknown position of the end-effector is described by the position vector $\mathbf{e}$, which defines the location of $\mathrm{E}$ at the distal end of the output link in the $\mathrm{F}(x, y, z)$ coordinate frame.

The first step to solve the direct kinematics of this specific manipulator consists in finding the intersection points of three spherical surfaces. The surface of each sphere represents the range of motion of distal end of one of the limbs when point $B_{n}$ is located at a known position (determined from the geometry of the robot and the angle given by the appropriate actuator). The radius of each sphere is equivalent to length $d_{2}$ and the intersection points of the three sphere surfaces are the only possible positions that point M may occupy.

In the general case, there are two intersection points, but other cases can be identified such as (1) a singular solution, where one sphere is tangent to the circle of intersection of the two other spheres, or (2) no solution, where no intersection point exists.

The equation of the sphere generated by the $n$th limb is given by:

$\left(m_{x}-b_{n x}\right)^{2}+\left(m_{y}-b_{n x}\right)^{2}+\left(m_{z}-b_{n x}\right)^{2}=d_{2}^{2}$

that can be extended to:

$m_{x}^{2}+m_{y}^{2}+m_{z}^{2}+m_{x} a_{1 n}+m_{y} a_{2 n}+m_{z} a_{3}+a_{4}=d_{2}^{2}$,

for $\quad n=1,2,3$

where:

$$
\begin{aligned}
& a_{1 n}=-2 d_{2} \cos \vartheta_{n} \cos \alpha_{n} \\
& a_{2 n}=-2 d_{2} \sin \vartheta_{n} \cos \alpha_{n}, \quad \text { for } n=1,2,3 \\
& a_{3 n}=-2 d_{2} \sin \alpha_{n} \\
& a_{4 n}=d_{2}^{2}
\end{aligned}
$$

The intersection of the two spheres, generated by limb 1 with limbs 2 and 3, creates two circles that are contained in the following plane:

$$
c_{1 n} m_{x}+c_{2 n} m_{y}+c_{3 n} m_{z}=0, \text { for } n=2,3
$$

where:

$$
\begin{aligned}
& c_{1 n}=a_{11}-a_{1 n} \\
& c_{1 n}=a_{21}-a_{2 n}, \text { for } n=2,3 \\
& c_{1 n}=a_{31}-a_{3 n}
\end{aligned}
$$


A line is then generated by intercepting the two planes. This may be represented by solving Eq. 10 for $m_{y}$ and $m_{z}$ as a function of $m_{x}$ :

$$
m_{y}=h_{1} m_{x} \text { and } m_{z}=h_{2} m_{x}
$$

where:

$$
\begin{aligned}
& h_{1}=\frac{-c_{12} c_{33}+c_{32} c_{13}}{-c_{32} c_{23}+c_{22} c_{33}} \\
& h_{2}=\frac{-c_{22} c_{13}-c_{12} c_{23}}{c_{32} c_{23}-c_{22} c_{33}}
\end{aligned}
$$

The intersections of this line with one of the spheres presented in Eq. 9, define the position of point $\mathrm{M}$. Therefore, introducing Eq. 11 on Eq. 9, for $n=1$ the following expression can be obtained:

$$
g_{1} m_{x}^{2}+g_{2} m_{x}+g_{3}=0
$$

where:

$$
\begin{aligned}
& g_{1}=1+h_{1}^{2}+h_{2}^{2} \\
& g_{2}=a_{11}+a_{12} h_{1}+a_{31} h_{2} \\
& g_{3}=-d_{2}^{2}+d_{1}^{2}
\end{aligned}
$$

and solving for $m_{x}$ :

$$
m_{x}=\frac{-g_{2}+\sqrt{g_{2}^{2}-4 g_{1} g_{3}}}{2 g_{1}}
$$

Having calculated the coordinates of point $M$, the end-effector coordinates are obtained by:

$$
\mathbf{e}=-\frac{\mathbf{m}}{\|\mathbf{m}\|}(\|\mathbf{I}\|-\|\mathbf{m}\|)
$$

which solves the direct kinematics problem for this manipulator.

\section{Velocity analysis}

Since the inverse kinematics equations for the Dionis manipulator are relatively complex, it is not computationally efficient to calculate the Jacobian Matrix, differentiating those relationships with respect to $x, y$ and $z$. Instead of this, we propose to obtain the velocity of the end effector, $v_{E}$ by deriving Eq. 3 with respect to time:

$$
\mathbf{v}_{E}=\mathbf{v}_{M}-\left(\frac{\mathbf{m}}{\|\mathbf{m}\|}\|\mathbf{I}\|\right)^{\prime}
$$

where:

$$
\mathbf{v}_{M}=\dot{\boldsymbol{\alpha}}_{n} \times \mathbf{d}_{1 n}+\left(\dot{\boldsymbol{\beta}}_{n}+\dot{\boldsymbol{\gamma}}_{n}\right) \times \mathbf{d}_{2 n}
$$

Multiplying both sides of Eq. 15 by $\mathbf{d}_{2 n}$, in the $L_{n}$ coordinate frame yields:

$$
{ }^{L_{n}} \mathbf{d}_{2 n} \cdot{ }^{L_{n}} \mathbf{v}_{M}=\dot{\alpha}_{n} \cdot\left({ }^{L_{n}} \mathbf{d}_{1 n} \times{ }^{L_{n}} \mathbf{d}_{2 n}\right)
$$

where:

$$
\begin{aligned}
& { }^{L_{n}} \mathbf{d}_{2 n}=\mathbf{d}_{2}\left[\begin{array}{c}
\sin \gamma_{n} \cos \left(\alpha_{n}+\beta_{n}\right) \\
\cos \gamma_{n} \\
\sin \gamma_{n} \sin \left(\alpha_{n}+\beta_{n}\right)
\end{array}\right], \\
& { }^{L_{n}} \mathbf{d}_{1 n}=\mathbf{d}_{1}\left[\begin{array}{c}
\cos \alpha_{n} \\
0 \\
\sin \alpha_{n}
\end{array}\right],{ }^{L_{n}} \dot{\boldsymbol{\alpha}}_{n}=\left[\begin{array}{c}
0 \\
-\dot{\alpha}_{n} \\
0
\end{array}\right] \\
& { }^{L_{n}} \mathbf{v}_{M}={ }^{L_{n}} R_{F}{ }^{F} \mathbf{v}_{M}=\left[\begin{array}{ccc}
\cos \vartheta_{n} & \sin \vartheta_{n} & 0 \\
-\sin \vartheta_{n} & \cos \vartheta_{n} & 0 \\
0 & 0 & 1
\end{array}\right]\left[\begin{array}{c}
v_{M x} \\
v_{M y} \\
v_{M z}
\end{array}\right] \\
& =\left[\begin{array}{c}
v_{M x} \cos \vartheta_{n}+v_{M y} \sin \vartheta_{n} \\
-v_{M x} \sin \vartheta_{n}+v_{M y} \cos \vartheta_{n} \\
v_{M z}
\end{array}\right]
\end{aligned}
$$

Equation 16 results in three scalar equations which can be arranged as follows:

$$
J_{x} \mathbf{v}_{M}=J_{\mathbf{q}} \dot{\mathbf{q}}
$$

where the direct and inverse kinematics Jacobian matrices are respectively:

$$
J_{x}=\left[\begin{array}{c}
\mathbf{j}_{1}^{T} \\
\mathbf{j}_{2}^{T} \\
\mathbf{j}_{3}^{T}
\end{array}\right], J_{\mathbf{q}}=\left[\begin{array}{ccc}
b_{1} & 0 & 0 \\
0 & b_{2} & 0 \\
0 & 0 & b_{3}
\end{array}\right]
$$

with:

$$
\begin{aligned}
\mathbf{j}_{n}= & {\left[\begin{array}{c}
\cos \left(\alpha_{n}+\beta_{n}\right) \sin \gamma_{n} \cos \vartheta_{n}-\cos \gamma_{n} \sin \vartheta_{n} \\
\cos \left(\alpha_{n}+\beta_{n}\right) \sin \gamma_{n} \sin \vartheta_{n}-\cos \gamma_{n} \cos \vartheta_{n} \\
\cos \left(\alpha_{n}+\beta_{n}\right) \sin \gamma_{n}
\end{array}\right], } \\
& \text { for } \quad n=1,2,3 \\
b_{n}= & a \sin \beta_{n} \sin \gamma_{n}, \quad \text { for } n=1,2,3
\end{aligned}
$$

and

$$
\dot{\mathbf{q}}=\left[\begin{array}{c}
\dot{\alpha}_{1} \\
\dot{\alpha}_{2} \\
\dot{\alpha}_{3}
\end{array}\right]
$$


Equation 17 can be written in a compact form by introducing the (overall) Jacobian matrix $\mathrm{J}$ :

$$
J \mathbf{v}_{M}=\dot{\mathbf{q}}
$$

\section{Singular configuration}

The identification of singular configurations is an important issue that must be addressed at the first stages of mechanism design. This topic has been studied for a long time [16] and comprehensive classifications have been proposed in past years [35]. The most remarkable cases are usually called (1) inverse kinematics singularities, when an infinitesimal motion of a limb does not yield a motion of the platform (that "loses" one or more DOF in certain directions) and (2) direct kinematics singularities, when the moving platform can move along certain directions even if all actuators are completely locked (and the mechanism "gains" one or more DOF). From Eq. 14 we may obtain that:

$$
\mathbf{v}_{E}=f\left(\mathbf{m}, \mathbf{v}_{M}\right)
$$

Introducing Eq. 17 on Eq. 19 we establish:

$$
\mathbf{v}_{E}=f\left(\mathbf{m}, J_{x}^{-1} J_{q} \dot{\mathbf{q}}\right)
$$

As a consequence, the singularity analysis of the Dionis manipulator can be performed considering $J_{x}$ and $J_{q}$ calculated in the previous section. The first kind of singularity occurs when $J_{q}$ is not of full rank and

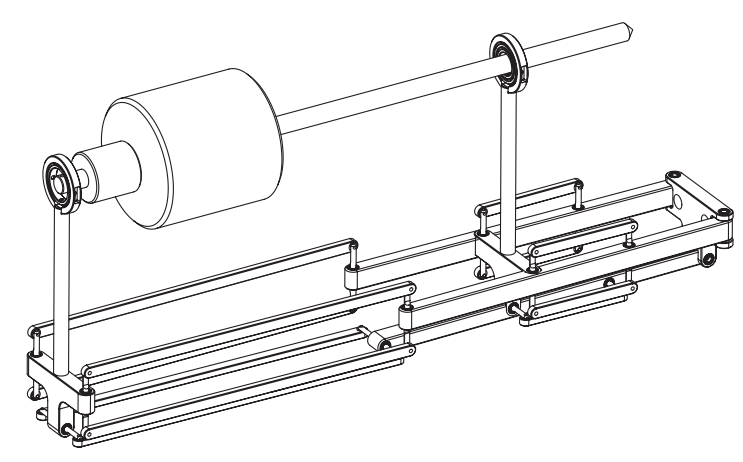

(a)

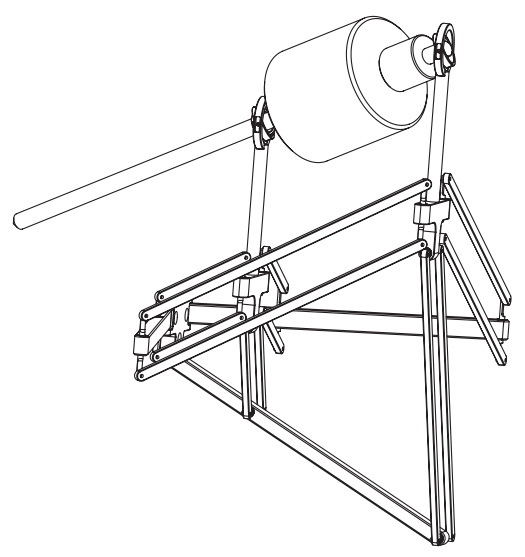

(c)

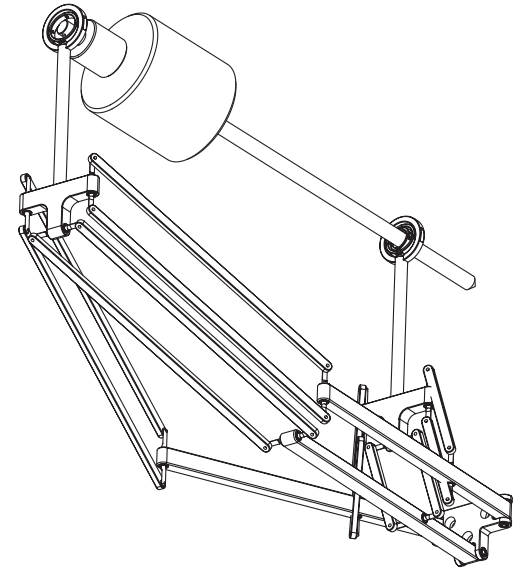

(b)

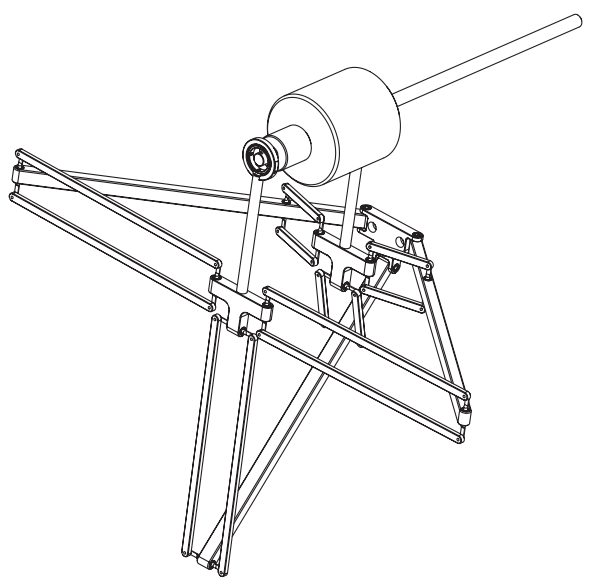

(d)

Fig. 11. Example of singular configurations. 
$J_{x}$ is invertible, i.e., $\operatorname{det}\left(J_{q}\right)=0$ and $\operatorname{det}\left(J_{x}\right) \neq 0$. For this manipulator, this is verified when:

$$
\alpha_{n}=0 \text { or } \alpha_{n}=\pi \text { or } \gamma_{n}=0 \text { or } \gamma_{n}=\pi \text {, for } n=1,2 \text { or } 3
$$

The second kind of singularity, named the direct kinematics singularity, occurs when $J_{x}$ is not of full rank while $J_{q}$ is invertible, i.e., $\operatorname{det}\left(J_{q}\right) \neq 0$ and $\operatorname{det}\left(J_{x}\right)=0$, which can happen when:

$$
\alpha_{n}+\beta_{n}=0 \text { or } \pi \text { or } \gamma_{n}=0 \text { or } \pi, \quad \text { for } n=1,2 \text { or } 3
$$

A third kind of singularity occurs when both $J_{q}$ and $J_{x}$ become simultaneously not invertible, i.e., $\operatorname{det}\left(J_{q}\right)=0$ and $\operatorname{det}\left(J_{x}\right)=0$ which happens when:

$$
\gamma_{n}=0 \text { or } \pi, \quad \text { for } n=1,2 \text { or } 3
$$

To summarize, singularities can occur when:

- all the pairs of the bars composing the parallelograms are parallel - the moving platforms have three degrees of freedom and move along a spherical surface rotating about an axis perpendicular to the platforms, Fig. 11(a).

- two pairs of bars composing the parallelograms, for each moving platform, are parallel - the moving platforms have one degree of freedom, moving in only one direction Fig. 11(b).

- two pairs of bars composing the parallelograms are in the same plane or in parallel planes - the moving platforms have only one degree of freedom, rotating about a vertical axis, Fig. 11(c).

- three parallelograms, of each moving platform, are placed at three parallel planes or on the same plane - the platforms keep three DOFs, namely: Two rotations about axes contained in the plane of the platform and one translation perpendicular to the same plane, Fig. 11(d).

\section{Workspace analysis}

Workspace is one of the most important issues when designing a parallel manipulator since it determines the region that can be reached and, therefore, it is a key point in robotic mechanism design [16]. The designs based on a workspace calculation use methods

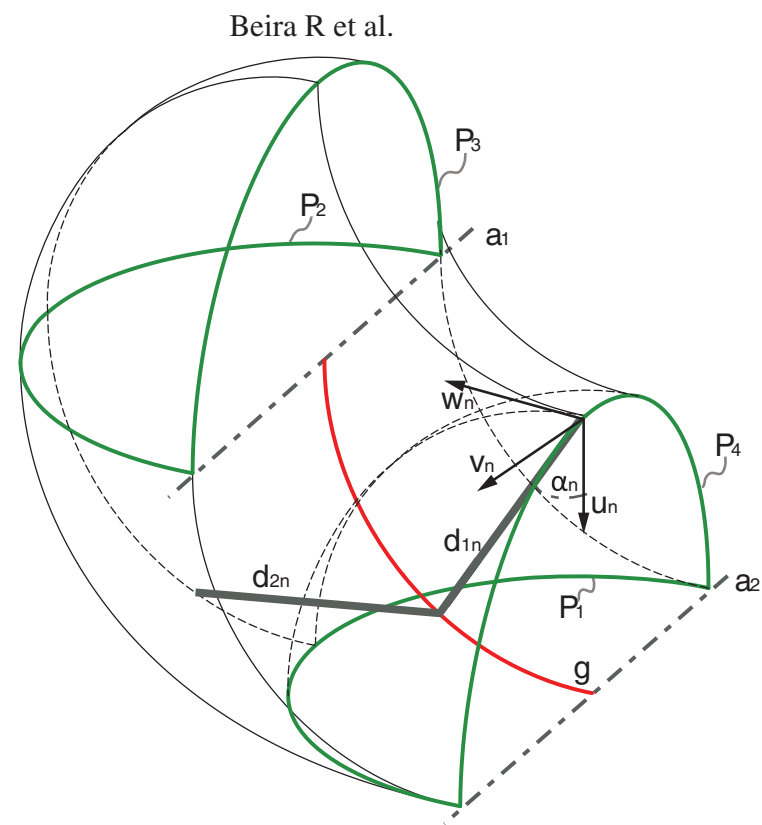

Fig. 12. Profiles generating the $n$th limb workspace.

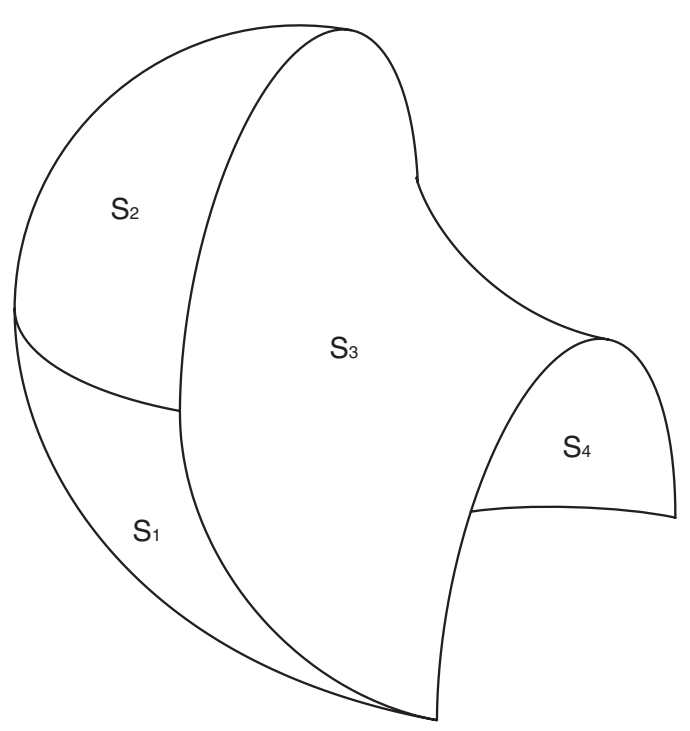

Fig. 13. Workspace surfaces for each limb.

in which the first step is to develop an objective function that might be reached by the result. The result is generally obtained by recursive-numerical-algorithms [35]. These methodologies have the disadvantage of being extremely time consuming, due to the highly non-linear objective functions that are manipulated. In 


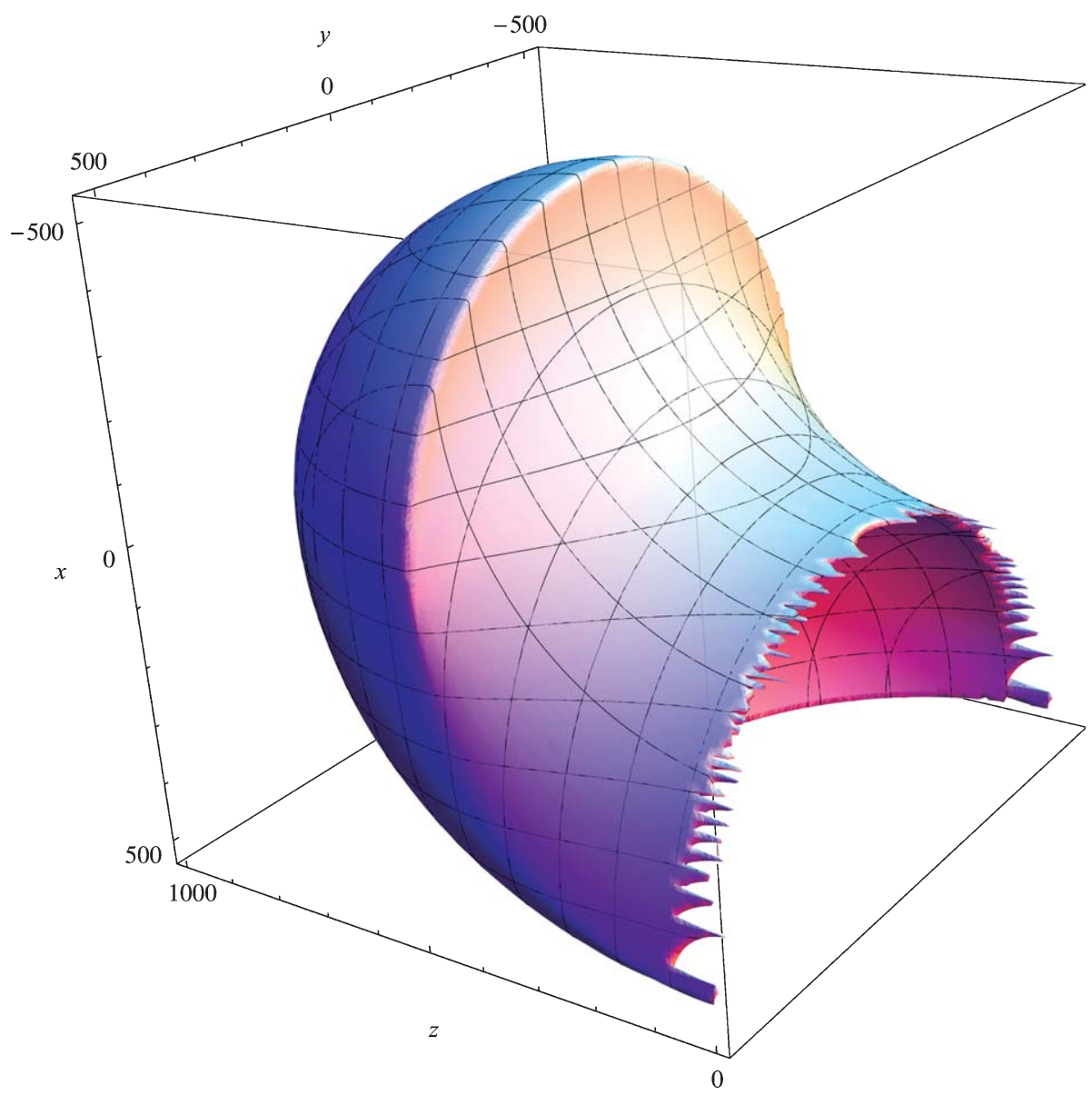

Fig. 14. 3D representation of the workspace of point $\mathrm{M}$, for a single limb (example with $d=500$ and $l=1000$ is shown).

this paper, the workspace representation of the Dionis manipulator is analysed geometrically.

\subsection{Characteristics of the workspace for a single limb}

Knowing in advance all the singular configurations presented in the previous section, it is possible to introduce some constraints in the manipulator's design in order to avoid those postures and collisions between mechanism components. Therefore, it was decided to analyze the workspace of the Dionis manipulator in the boundary of those conditions, where $\alpha_{n} \in[0, \pi / 2]$, $\gamma_{n} \in[0, \pi]$ and $d_{1}=d_{2}=d$.

Equation 3 indicates that, for a given position of the moving point $\mathrm{M}$, the position of the end-effector, $\mathrm{E}$, can be determined by a translation through vector $\mathbf{l}$. In other words, the workspace generated by the $n$th limb is a translation of the reachable workspace of point $\mathrm{M}$ by $\mathbf{l}$. In addition, the motion of the limb is constrained, not only by the joint limits, but also by the other limbs. Therefore, the workspace of Dionis Manipulator is the intersection of the three individual reachable workspaces generated by the three limbs.

According to Eq. 8, and the specific Dionis limb design, the workspace of the limb point $\mathrm{M}$ is a solid sphere with radius $d$, if there is no joint limitations for the revolute joints. However, point $B_{n}\left(b_{n x}, b_{n y}, b_{n z}\right)$, which is able to move along a circular path in the ZX plan, is limited to avoid singular configurations and colisions with other components of the mechanism. The workspace of each limb is the solid envelope shown in Fig. 12.

In order to calculate and visualize the workspace for each limb, the following steps have to be achieved: 


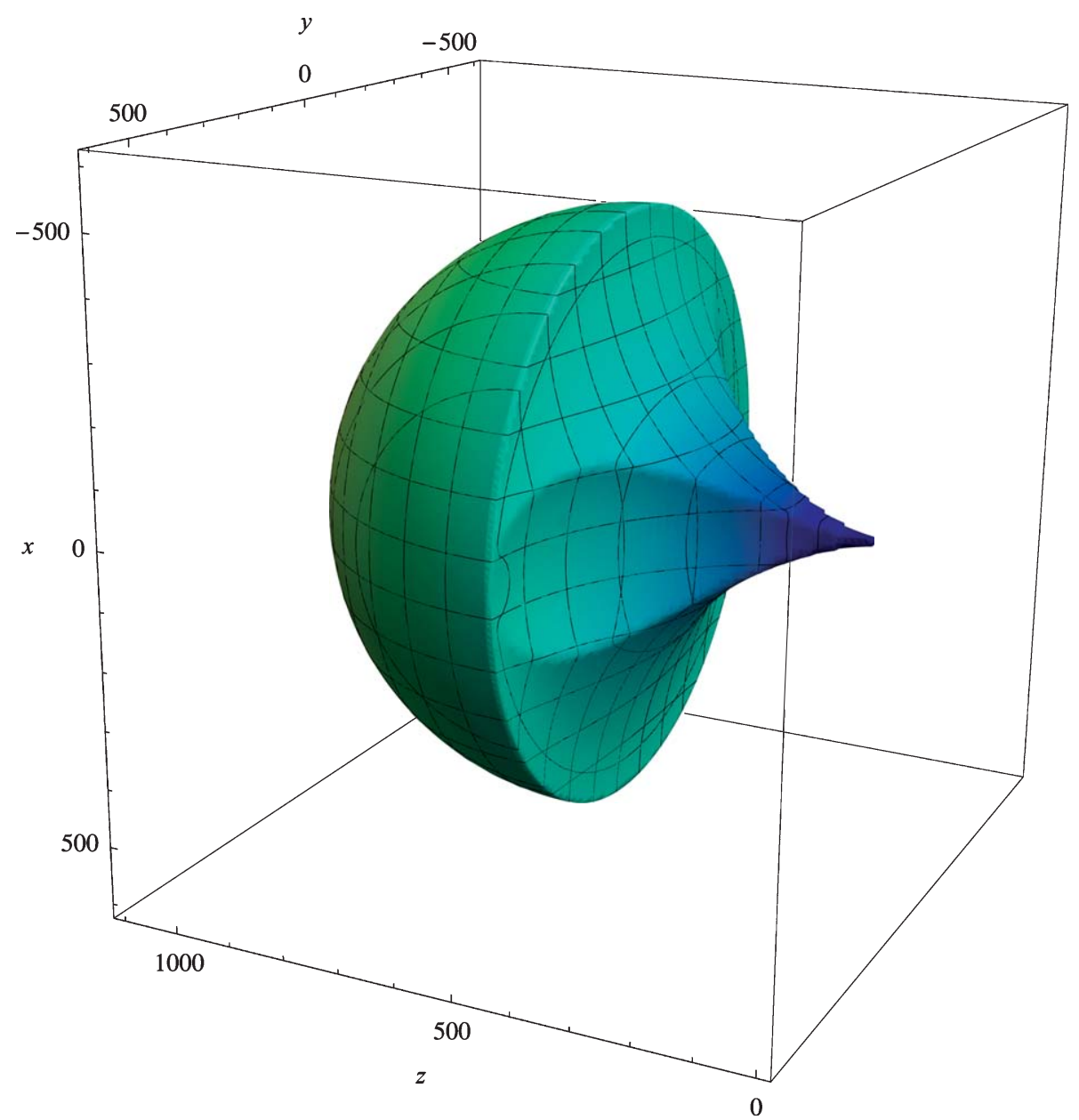

Fig. 15. 3D representation of the workspace of point $M$ (example with $\vartheta_{1}=0 \mathrm{rad}, \vartheta_{2}=\pi / 2$ and $\vartheta_{3}=-\pi / 2 \mathrm{rad}$ ).

(1) parallel translation of profile $p_{1}$ (generated by the path of point $\mathrm{M}$ at full range of $\gamma_{n}$, for $\alpha_{n}=0$ ) along the guide-line $g$, until $p_{2}$;

(2) rotation of profile $p_{2}$ along axis $a_{1}$, until $p_{3}$;

(3) parallel translation of profile $p_{3}$ along the guideline $g$, until $p_{4}$

(4) rotation of profile $p_{4}$ along axis $a_{2}$, until $p_{1}$ again.

The corresponding analytical equations of the above mentioned profiles and guide lines are the following:

$$
\begin{aligned}
& P_{1}: u_{n}=d \wedge v_{n}^{2}+w_{n}^{2}=d^{2} \\
& P_{2}: u_{n}=0 \wedge v_{n}^{2}+\left(w_{n}-d\right)^{2}=d^{2}
\end{aligned}
$$

$$
\begin{aligned}
& P_{3}: w_{n}=d \wedge u_{n}^{2}+v_{n}^{2}=d^{2} \\
& P_{4}: w_{n}=0 \wedge\left(u_{n}-d\right)^{2}+v_{n}^{2}=d^{2} \\
& g: v_{n}=0 \wedge u_{n}^{2}+z^{2}=d^{2} \\
& a_{1}: w_{n}=d \wedge u_{n}=0 \\
& a_{2}: w_{n}=0 \wedge u_{n}=d
\end{aligned}
$$

With the profiles presented before, it is possible to generate the surfaces shown in Fig. 13.

The resulting surfaces, $s_{i}(i=1,4)$ in Fig. 13 are generated from the following equations: 


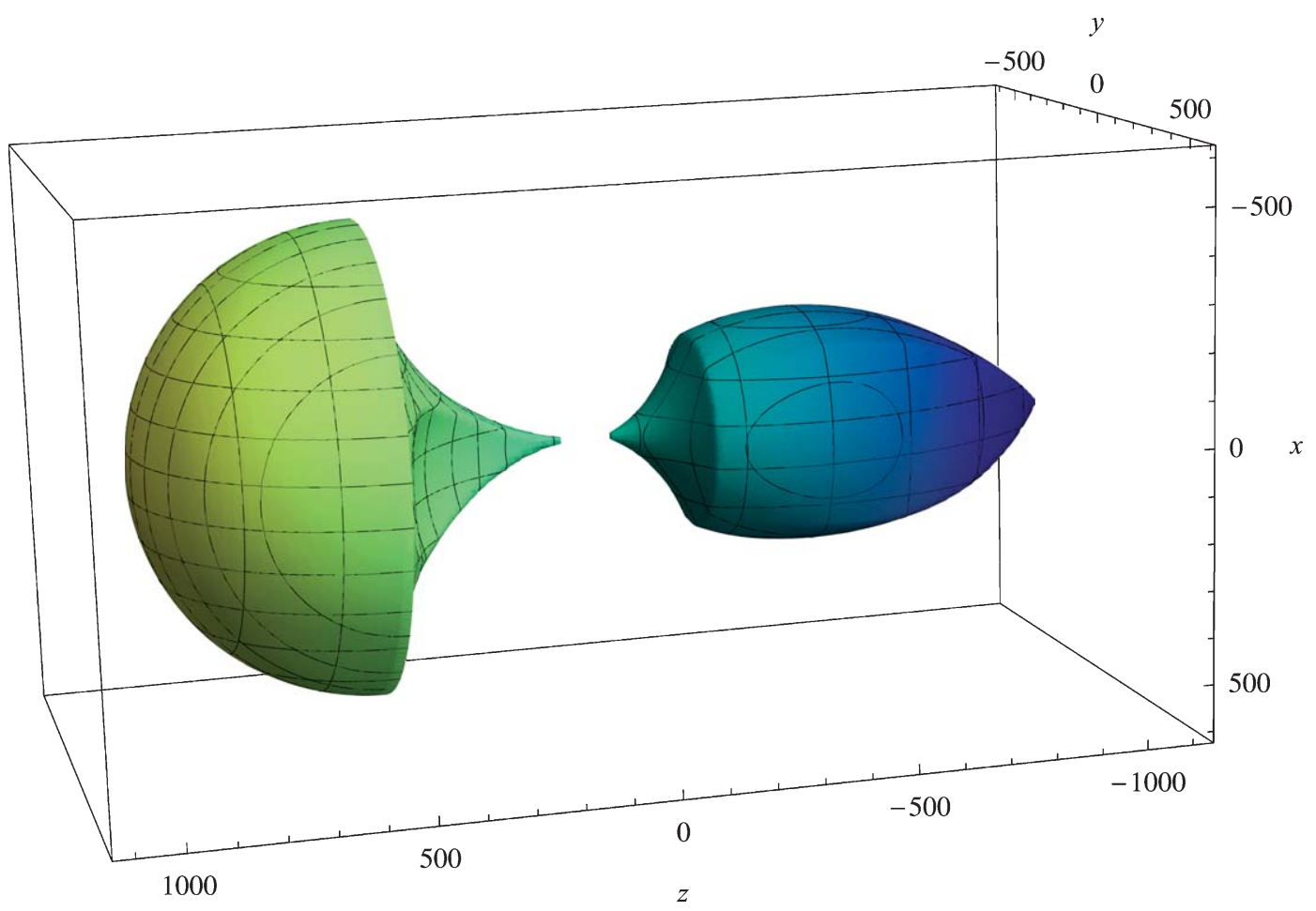

Fig. 16. 3D representation of the workspace of points $\mathrm{M}(z>0)$ and $\mathrm{E}(z<0)$.

$$
\begin{aligned}
& s_{1}: u_{n}^{2}+\left(w_{n}-\sqrt{d^{2}-v_{n}}\right)^{2}=d^{2} \\
& s_{2}: u_{n}^{2}+v_{n}^{2}+\left(w_{n}-d\right)^{2}=d^{2} \\
& s_{3}: u_{n}^{2}+\left(v_{n}-\sqrt{d^{2}-w_{n}}\right)^{2}<d^{2} \\
& s_{4}: u_{n}^{2}+\left(v_{n}-d\right)^{2}+w_{n}^{2}=d^{2}
\end{aligned}
$$

Once the former analytical expressions have been identified, it is possible to represent them in the $3 \mathrm{D}$ space, using Wolfram Mathematica 7 , and visualize the workspace of the manipulator, as in Fig. 14.

The workspace of $\mathrm{M}$, considering the entire manipulator, is the result of the intersection of the workspaces of the 3 limb workspaces, Fig. 15.

Having the Workspace of point $\mathrm{M}, W_{M}$, defined, the workspace of E, $W_{E}$, is calculated using Eq. 13, Fig. 16. On the left part of the plot, for $z>0$, we may find the workspace of $\mathrm{M}$, while the workspace of $\mathrm{E}$ is represented for $z<0$. As can be seen by the workspace distribution around point $\mathrm{O}(0,0,0)$, the stationary of the mechanism's RCM is verified. The reachable workspace of the Dionis Manipulator can be represented easily using the commercial CAD software such as SolidWorks 2009. For instance, in the example with $d=500, l=1000, \vartheta_{1}=0 \mathrm{rad}$ and $\vartheta_{2}=\pi / 2$, $\vartheta_{3}=-\pi / 2 \mathrm{rad}$, the workspace has the shape shown on Fig. 17. It can be seen that it encloses the patients abdominal cavity, meeting the specifications in terms of task workspace, for MIS.

\section{Conclusions}

The major focus of this work is to present a novel concept of external positioning manipulator for minimally invasive surgical procedures. The proposed manipulator provides enough dexterity and precision needed to position MIS instruments at any location within the abdominal cavity. The implementation of a unique parallel kinematics results in a 4-DOF hybrid mechanism that provides three rotations and one translation, with a fixed remote center of motion. A significant advantage of this novel design is that it can be placed below the operation table plane, allowing direct access to the patient without removing the manipulator. Consequently, safety is improved and considerable space in the operating room is saved. The latter features 


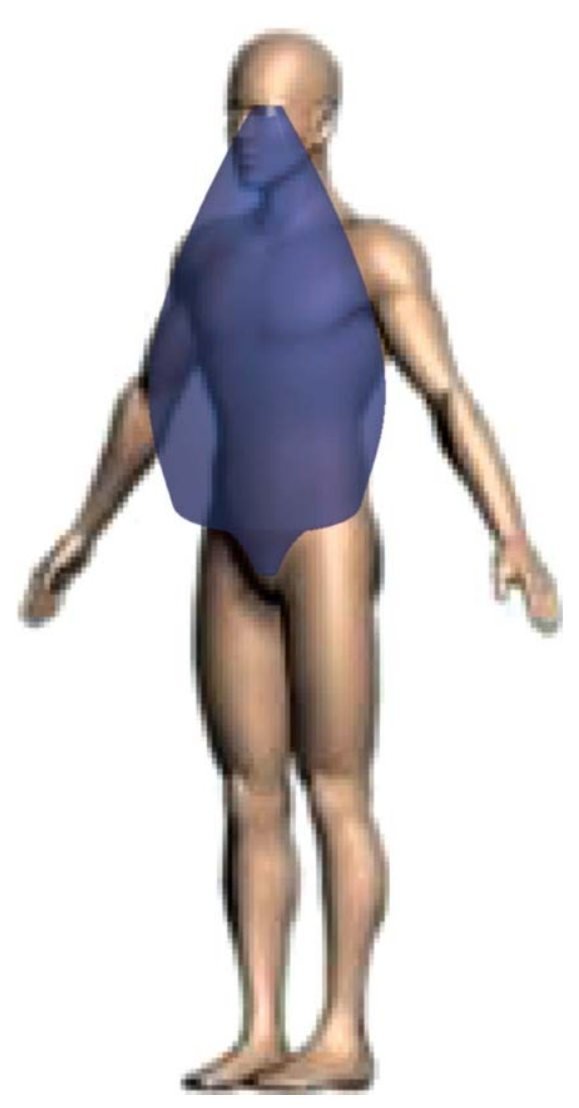

Fig. 17. Workspace with respect to patient.

prove the interest of the proposed design in comparison to existing solutions. In addition, compactness, simplicity and robustness make the Dionis Manipulator a highly qualified candidate for MIS procedures.

Analysis of direct and inverse kinematics carried out in close form demonstrate the large simplicity of the Dionis Manipulator. Moreover, analytical conditions to identify singular configurations of the mechanism have been studied and geometrical interpretation provided. Results indicate that singularities may be removed from the workspace with careful design of the mechanical system. A geometry-based workspace analysis was formulated, consisting in a simple and applicable tool to design manipulators as the one presented in this work. The specific surgical workspace was verified, confirming the proposed mechanism suitability for MIS procedures.

Further optimization is necessary for both stiffness and dynamic performance of the Dionis design. In addition, clinical use needs to be considered in future work to demonstrate the applicability of this highly promising surgical manipulator.

\section{Acknowledgements}

The authors are much indebted to surgeons Monica Hagen, Oliver Wagner and Professor Philippe Morel (HUG, Switzerland) for the fruitful and in depth discussions on advanced surgical techniques and the useful contacts they provided. We also gratefully acknowledge the support of the IST-EPFL Joint Scholarship initiative and to the ARAKNES FP7 European Project EU/IST-2008-224565.

\section{References}

[1] I. Agricola and T. Friedrich, Elementary geometry. Illustrated ed., AMS Bookstore, vol. 43 of Student mathematical library, 2008

[2] R. Bailey, K. Zucker, J. Flowers, W. Scovill, S. Graham and A. Imbembo, Laparoscopic cholecystectomy. Experience with 375 consecutive patients, Annals of surgery 214(4) (1991), 531; Lippincott, Williams, and Wilkins.

[3] M. Barbosa, F. Nahas and L. Ferreira, A practical dressing to the umbilical stalk, Journal of Plastic, Reconstructive \& Aesthetic Surgery, Vol. 61, 2008; Elsevier.

[4] E. Bass, H. Pitt and K. Lillemoe, Cost-effectiveness of laparoscopic cholecystectomy versus open cholecystectomy, American Journal of Surgery 165(4) (1993), 466.

[5] R. Baumann, Haptic interface for virtual reality based laparoscopic surgery training environment, Unpublished doctoral dissertation 1734, (1997).

[6] P. Berkelman, E. Boidard, P. Cinquin and J. Troccaz, LER: The light endoscope robot, IEEE/RSJ International Conference on Intelligent Robots and Systems (IROS). Proceedings 3, 2003.

[7] P. Berkelman and J. Ma, A compact, modular, teleoperated robotic minimally invasive surgery system. Biomedical Robotics and Biomechatronics. BioRob. The First IEEE/RASEMBS International Conference on, 2006

[8] M. Cavusoglu, F. Tendick, M. Cohn and S. Sastry, A laparoscopic telesurgical workstation, IEEE Transactions on Robotics and automation 15(4) (1999), 728-739.

[9] R. Chamberlain and S. Sakpal, A comprehensive review of single-incision laparoscopic surgery (SILS) and natural orifice transluminal endoscopic surgery (NOTES) techniques for cholecystectomy, Journal of Gastrointestinal Surgery 13(9) (2009), 1733-1740; Springer.

[10] R. Clavel, Delta, a Fast Robot with Parallel Geometry, Vol. 26-28, Springer-Verlag and Heidelberg GmbH and Co., Berlin, 1988, pp. 91-100.

[11] J. Cugura, I. Kirac, T. Kulis, J. JankoviT, M. Beslin, First case of single incision laparoscopic surgery for totally extraperitoneal inguinal hernia repair, Acta Clin Croat 47(4) (2008), 249.

[12] P.G. Curcillo Ii, S.A. King, E.R. Podolsky and S.J. Rottman, Single Port Access (SPA) minimal access surgery through a single incision, Surg Technol Int 18 (2009), 19-25. 
[13] L. de Sousa, J. deSousa, L. deSousa Filho, M. de Sousa, V. deSousa, A. de Sousa, R. Zorron, Totally NOTES (T-NOTES) transvaginal cholecystectomy using two endoscopes: Preliminary report, Surgical Endoscopy Springer, 2009, pp. 1-6.

[14] G. Dini, L. Ferreira, A simple technique to correct umbilicus vertical malposition, Plastic and Reconstructive Surgery 119(6) (2007), 1973.

[15] M. Gettman, G. Box and T. Averch, Consensus statement on natural orifice transluminal endoscopic surgery and single-incision laparoscopic surgery: Heralding a new era in urology? European Urology 53(6) (2008), 1117-1120; Elsevier.

[16] C. Gosselin and J. Angeles, Singularity analysis of closedloop kinematic chains, IEEE Transactions on Robotics and Automation 6(3) (1990), 281-290.

[17] A. Guerrouad and P. Vidal, SMOS: Stereotaxical microtelemanipulator for ocular surgery. Engineering in Medicine and Biology Society, 1989. Images of the Twenty-First Century, Proceedings of the Annual International Conference of the IEEE Engineering, 1989, pp. 879-880.

[18] P. Guillou, P. Quirke, H. Thorpe, J. Walker, D. Jayne, A. Smith, R. Heath, J. Brown, Short-term endpoints of conventional versus laparoscopic-assisted surgery in patients with colorectal cancer (MRC CLASICC trial): multicentre, randomised controlled trial, The Lancet 365(9472) (2005), 1718-1726; Elsevier.

[19] G. Guthart and K. Salisbury, The inituitive telesurgical system: Overview and application, Proceedings of the 2000 IEEE ICRA, 2000, pp. 618-621.

[20] M. Hagen, O. Wagner, D. Christen and P. Morel, Cosmetic issues of abdominal surgery: results of an enquiry into possible grounds for a natural orifice transluminal endoscopic surgery (NOTES) approach, Endoscopy 40(7) (2008), 581583; Georg Thieme Verlag, Stuttgart, 1969-.

[21] M. Hagen, O. Wagner, K. Thompson, G. Jacobsen, A. Spivack, B. Wong, M. Talamini, S. Horgan, Supra-pubic single incision cholecystectomy, Journal of Gastrointestinal Surgery (2009), 1-4; Springer.

[22] G.J. Hamlin and A.C. Sanderson, A novel concentric multilink spherical joint with parallel robotics applications, IEEE International Conference on Robotics and Automation, Vol. 2, San Diego, CA, 1994, pp. 1267-1272.

[23] M. Lum, J. Rosen, M. Sinanan and B. Hannaford, Kinematic optimization of a spherical mechanism for a minimally invasive surgical robot, IEEE International Conference on Robotics and Automation, Vol. 1, 2004, pp. 829-834.

[24] M. Lum, J. Rosen, M. Sinanan, B. Hannaford, Citeseer; Optimization of a spherical mechanism for a minimally invasive surgical robot: theoretical and experimental approaches. IEEE Transactions on Biomedical Engineering 53(7) (2006), 1440_ 1445 .

[25] A. Madhani, G. Niemeyer, J. Salisbury Jr, The Black Falcon: a teleoperated surgical instrument for minimallyinvasive surgery, IEEE/RSJ International Conference on Intelligent Robots and Systems. Proceedings Vol 2, 1998.

[26] F. Montz, C. Holschneider, M. Munro, Incisional hernia following laparoscopy: a survey of the American Association of Gynecologic Laparoscopists, Obstetrics \& Gynecology 84(5) (1994), 881.

[27] J. Perissat, D. Collet, R. Belliard, J. Desplantez, E. Magne, Laparoscopic cholecystectomy: the state of the art. A report on 700 consecutive cases, World Journal of Surgery 16(6) (1992), 1074-1082; Springer

[28] J. Rosen, J. Brown, L. Chang, M. Barreca, M. Sinanan and B. Hannaford, The bluedragon-a system for measuring the kinematics and the dynamics of minimally invasive surgica tools in-vivo. Proceedings-IEEE International Conference on Robotics and Automation 2 (2002), 1876-1881.

[29] J. Sackier and Y. Wang, Robotically assisted laparoscopic surgery, Surgical endoscopy 8(1) (1994), 63-66; Springer.

[30] R. Taylor, J. Funda, B. Eldridge, S. Gomory, K. Gruben D. LaRose, Talamini M, L. Kavoussi, J. Anderson, A telerobotic assistant for laparoscopic surgery, IEEE Engineering in Medicine and Biology Magazine 14(3) (1995), 279-288.

[31] L. Tsai, Robot analysis: the mechanics of serial and parallel manipulators, Wiley-Interscience, 1999.

[32] M. Turner, D. Perkins, A. Murray and P. Larochelle, Systematic process for constructing spherical four-bar mechanisms, ASME International Mechanical Engineering Congress and Exposition, 2005.

[33] P. Vischer and R. Clavel, Multimedia Archives; Argos: a novel 3-DoF parallel wrist mechanism, The International Journal of Robotics Research, 19(1) (2000), 5.

[34] N. Zemiti, T. Ortmaier, G. Morel, A new robot for force control in minimally invasive surgery. IEEE/RSJ International Conference on Intelligent Robots and Systems (IROS). Proceedings Vol 4, 2004

[35] D. Zlatanov, R. Fenton and B. Benhabib, Identification and classification of the singular configurations of mechanisms, Mechanism and Machine Theory 33(6) (1998), 743-760 Elsevier.

[36] R. Zorrón, M. Filgueiras, L.C. Maggioni, L. Pombo, G. Lopes Carvalho, A. Lacerda Oliveira, NOTES. Transvaginal cholecystectomy: report of the first case, Surg Innov 14(4) (2007), 279-283. 

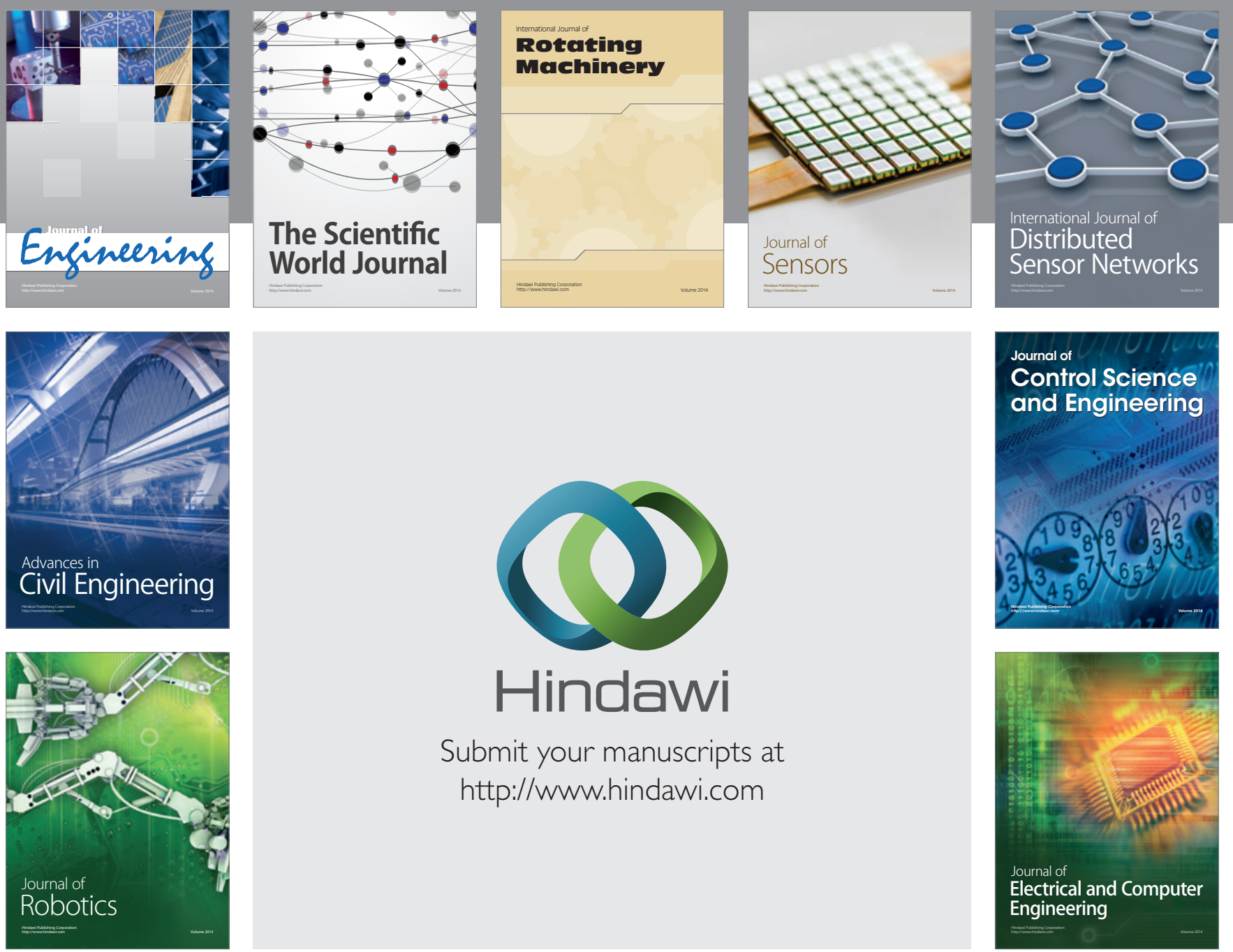

Submit your manuscripts at

http://www.hindawi.com
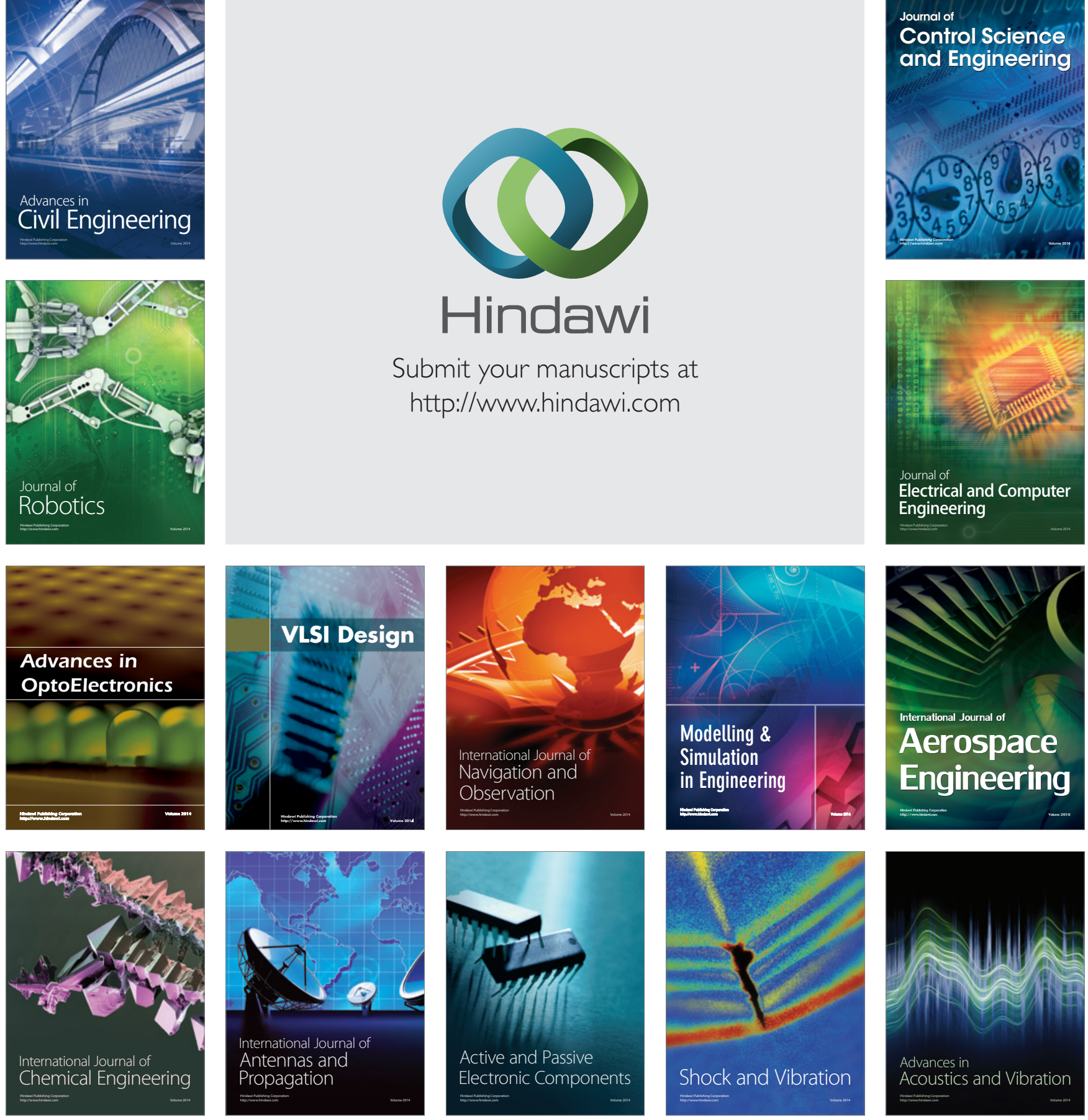